\title{
Zur Theorie der windschiefen Flächen.
}

(Von Herrn J. Lüroth in Mannbeim.)

\section{§. 1. Coordinaten einer geraden Linie.}

Sind $a_{1}, a_{2}, a_{3}, a_{4}$ und $b_{1}, b_{2}, b_{3}, b_{4}$ die homogenen Coordinaten zweier Punkte im Raum, so führen wir als Punktcoordinaten der durch diese Punkte bestimmten geraden Linie mit Plïcker (Phil. Trans. 1865) die Verhältnisse der sechs Grössen

$$
\begin{array}{lll}
a_{1} b_{2}-a_{2} b_{1}, & a_{1} b_{3}-a_{3} b_{1}, & a_{1} b_{4}-a_{4} b_{1}, \\
a_{3} b_{4}-a_{4} b_{3}, & a_{2} b_{4}-a_{4} b_{2}, & a_{2} b_{3}-a_{3} b_{2}
\end{array}
$$

ein. Wir sind hierzu berechtigt, weil diese Quotienten die wesentliche Eigenschaft der Coordinaten besitzen, dass sie ungeändert bleiben, wenn man die Elemente, von welchen sie abhängen, auf dem Gebilde, welches sie bestimmen, beliebig verlegt. In der That, setzen wir $a_{i}+\lambda b_{i}$ für $a_{i}, a_{i}+\mu b_{i}$ für $b_{i}$ so multipliren sich alle sechs Grössen mit dem Factor $(\mu-\lambda)$ und ihre Verhältnisse ändern sich nicht. Jene Grössen selbst werden wir homogene Coordinaten der Linie nennen und folgende Zeichen für sie gebrauchen:

$$
\text { (1.) }\left\{\begin{array}{lll}
x_{1}=a_{1} b_{2}-a_{2} b_{1}, & x_{2}=a_{1} b_{3}-a_{3} b_{1}, & x_{3}=a_{1} b_{4}-a_{4} b_{1}, \\
x_{4}=a_{3} b_{4}-a_{4} b_{3}, & x_{5}=a_{4} b_{2}-a_{2} b_{4}, & x_{6}=a_{2} b_{3}-a_{3} b_{2} .
\end{array}\right.
$$

Zwischen diesen sechs Coordinaten besteht die identische Gleichung

$$
\text { (2.) } x_{1} x_{4}+x_{2} x_{5}+x_{3} x_{6}=0,
$$

so dass sie nur vier unabhängige Grössen vertreten; wie dies sein muss, da eine Gerade durch vier Bedingungen bestimmt ist.

Sollen umgekehrt sechs Grössen $x_{1}, \ldots x_{6}$ Coordinaten einer geraden Linie sein, so müssen sich Grössen $a, b$ so bestimmen lassen, dass die Gleichungen (1.) befriedigt werden. Aus diesen folgen aber die Gleichungen

$$
\begin{array}{r}
-a_{2} x_{4}-a_{3} x_{5}-a_{4} x_{6}=0 \\
a_{1} x_{4}-a_{3} x_{3}+a_{4} x_{2}=0 \\
a_{1} x_{5}+a_{2} x_{3}-a_{4} x_{1}=0 \\
a_{1} x_{6}-a_{2} x_{2}+a_{3} x_{1}=0
\end{array}
$$

und damit diese zusammen bestehen können, muss ihre Determinante ver- 
schwinden. Diese ist eine überschlagene und das Quadrat von $x_{1} x_{4}+x_{2} x_{5}+x_{3} x_{6}$, was also Null sein muss.

Hiermit ist gezeigt, dass die Bedingung (2.) nothwendig und hinreichend dafür ist, dass sechs Grössen $x_{1}, \ldots x_{6}$ Coordinaten einer Geraden sind.

Sind andererseits $\alpha_{1}, \alpha_{2}, \alpha_{3}, \alpha_{4}, \beta_{1}, \beta_{2}, \beta_{3}, \beta_{4}$ die homogenen Coordinaten zweier Ebenen, so werden wir aus demselben Grunde wie oben die Grössen

$$
\begin{aligned}
& y_{1}=\alpha_{1} \beta_{2}-\alpha_{2} \beta_{1}, \quad y_{2}=\alpha_{1} \beta_{3}-\alpha_{3} \beta_{1}, \quad y_{3}=\alpha_{1} \beta_{4}-\alpha_{4} \beta_{1}, \\
& y_{4}=\alpha_{3} \beta_{4}-\alpha_{4} \beta_{3}, \quad y_{5}=\alpha_{4} \beta_{2}-\alpha_{2} \beta_{4}, \quad y_{6}=\alpha_{2} \beta_{3}-\alpha_{3} \beta_{2}
\end{aligned}
$$

als homogene Ebenencoordinaten der Schnittlinie beider Ebenen bezeichnen. Diese beiden Arten von Coordinaten einer geraden Linie sind jedoch nicht wesentlich von einander verschieden. Bezeichnen wir mit $\xi$ die laufenden Coordinaten, mit $p, q$ die Coordinaten zweier beliebigen Punkte, so sind die Gleichungen zweier Ebenen, welche durch $a$ und $b$ gehen

$$
\Sigma \pm \xi_{1} a_{2} b_{3} p_{4}=0, \quad \Sigma \pm \xi_{1} a_{2} b_{3} q_{4}=0 \text {. }
$$

Entnehmen wir hieraus die Coordinaten der Ebenen und bilden die Grössen $\boldsymbol{y}$, so finden wir mit Hülfe eines bekannten Satzes der Determinantentheorie, wenn $\Sigma \pm a_{1} b_{2} p_{3} q_{4}=D$ gesetzt wird

$$
y_{1}=D x_{4}, \quad y_{4}=D x_{1}, \quad y_{2}=D x_{5}, \quad y_{5}=D x_{2}, \quad y_{3}=D x_{6}, \quad y_{6}=D x_{3} .
$$

Es seien nun $x_{1}, \ldots x_{6}, y_{1}, \ldots y_{6}$ die Coordinaten zweier Geraden; wir wollen die Bedingung aufsuchen, unter der sie sich schneiden. Bezeichnen wir mit $a, b$ zwei Punkte der einen, mit $\alpha, \beta$ zwei Punkte der anderen Linie, so ist die Bedingung des Schnittes das Bestehen der Gleichungen

$$
a_{i}+\lambda b_{i}=\mu \alpha_{i}+\nu \beta_{i}, \quad(i=1,2,3,4)
$$

welches erfordert, dass

$$
\Sigma \pm a_{1} b_{2} \alpha_{3} \beta_{4}=0
$$

sei. Durch Entwickelung ergiebt sich hieraus die Bedingung

$$
\text { (3.) } \quad x_{1} y_{4}+x_{4} y_{1}+x_{2} y_{5}+x_{5} y_{2}+x_{3} y_{6}+x_{6} y_{3}=0 \text {. }
$$

Bezeichnen wir, wie im Folgenden stets,

$$
x_{1} x_{4}+x_{2} x_{5}+x_{3} x_{6} \text { mit } R,
$$

so schreibt sich die Gleichung (3.) auch

$$
\Sigma y_{i} \frac{\partial R}{\partial x_{i}}=0
$$


§. 2. Gebilde aus geraden Linien.

Damit sechs Grössen $x_{1}, \ldots x_{6}$ die Coordinaten einer geraden Linie seien, muss die Bedingung bestehen

$$
\boldsymbol{R}=\mathbf{0} \text {. }
$$

Sollen nun die Coordinaten noch einer Gleichung

$$
u=0
$$

genügen, wo $u$ eine homogene Function von $x_{1}, \ldots x_{6}$, so giebt es eine dreifach unendliche Schaar von Geraden, welche dieser Gleichung Genüge leisten. Das so erhaltene Gebilde heisst nach Pliicker ein Complex, und zwar vom Grade $n$, wenn $u$ eine Function $n^{\text {ter }}$ Ordnung ist. Die Function $u$ enthält dann

$$
\frac{n+1 . n+2 . n+3 . n+4 . n+5}{1.2 .3 .4 \cdot 5}=(n+5)_{5}
$$

Glieder und $(n+5)_{5}-1$ willkürliche Constanten, von welchen man noch, wenn $n \geqq 2$ ist, mit Hülfe der Gleichung $R=0(n+3)_{5}$ zerstören kann, so dass noch $\frac{n+1 \cdot(n+2)^{2} \cdot n+3}{3.4}-1$ übrig bleiben. Für $n=1$ stimmen beide Formeln überein, so dass man allgemein sagen kann:

Ein Complex $n^{\text {ter }}$ Ordnung wird durch $\frac{n+1 .(n+2)^{2} \cdot n+3}{3.4}-1$ Gerade bestimmt. Wenn Relationen zwischen den Coefficienten Statt haben, so kann der Complex übergehen in das System der Tangenten einer Oberfläche oder in das System der Geraden, welche eine Raumcurve schneiden.

Sollen die Coordinaten $x_{1}, \ldots x_{6}$ noch eine dritte Gleichung

$$
v=0
$$

erfüllen, so genügt nur noch eine zweifach unendliche Schaar von Geraden ' diesen Bedingungen. Man nennt die Gesammtheit dieser Linien nach Plücker eine Congruenz oder nach älterem Sprachgebrauch ein Strahlensystem.

Tritt zu den drei Gleichungen noch eine vierte

$$
w=0
$$

hïzu, so existirt nur eine einfach unendliche Schaar von geraden Linien, deren Coordinaten die vier Gleichungen erfüllen. Diese bilden eine Configuration oder im Allgemeinen eine windschiefe Fläche. Ordnung und Klasse der windschiefen Fläche sind gleich der Anzahl der Geraden, welche eine gegebene Gerade schneiden, also gleich der Zahl der gemeinsamen Wurzeln der Gleichungen

$$
\begin{gathered}
R=0, \quad u=0, \quad v=0, \quad w=0, \\
\Sigma a_{i} \frac{\partial R}{\partial x_{i}}=0,
\end{gathered}
$$


wo $a$ die Coordinaten einer beliebigen Linie sind. Ist $u$ von der $n^{\text {len }}$, $v$ von der $m^{\text {ten }}, w$ von der $l^{\text {ten }}$ Ordnung, so ist die Anzahl der gemeinsamen Wurzeln $2 \operatorname{lmn}$

und dies also die Ordnung und Klasse der Fläche, welche der Schnitt der drei Complexe $u=0, v=0, w=0$ ist.

§. 3. Das Geschlecht der windschiefen Fläche.

Denkt man sich die vier Gleichungen

$$
R=0, \quad u=0, \quad v=0, \quad w=0
$$

aufgelöst, so werden sich die Verhältnisse der sechs Coordinaten darstellen als, im Allgemeinen, algebraische Functionen eines Parameters. Statt dessen aber kann man sagen: die Coordinaten werden sich darstellen als ganze Functionen von zwei Parametern, welche durch eine Gleichung verknüpft sind. Diese Gleichung oder, wenn sie reductibel ist, einer ihrer irreductibeln Factoren bedingt dann eine gewisse Klasse von Abelschen Integralen und Functionen und zwar $2 p$ fach periodische, wenn $p$ die charakteristische Zahl der Abelschen Functionen ist, auf welche sie führt. Bekanntlich lassen sich dann jene Parameter durch solche Functionen darstellen, und das Gleiche gilt demnach von den Coordinaten einer Erzeugenden derjenigen einfachen windschiefen Fläche, welche dem gewählten irreductibeln Factor entspricht. Wir werden Geschlecht der Fläche die charakteristische Zahl der Abelschen Functionen nennen, auf welche sie führt, und stellen uns jetzt die Aufgabe, dieses zu bestimmen. Um die Coordinaten $x_{1}, \ldots x_{6}$ als Functionen zweier Parameter in der angegebenen Weise darzustellen, verbinden wir mit den Gleichungen:

$$
R=0, \quad u=0, \quad v=0, \quad w=0
$$

noch zwei Büschel von linearen Complexen

$$
\begin{aligned}
& a+s b=0, \\
& \alpha+z \beta=0,
\end{aligned}
$$

wo $a, b, \alpha, \beta$ lineare Ausdrücke der $x$ sind, und eliminiren aus diesen sechs Gleichungen die Coordinaten. Die resultirende Gleichung oder einer ihrer irreductibeln Factoren

$$
\varphi(s, z)=0
$$

ist dann die .Gleichung, deren $p$ wir suchen. Ist $k$ der Grad der Gleichung, welche in $s$ und $z$ von gleichem Grade ist, $\omega$ die Anzahl ihrer Verzweigungspunkte, so ist nach Riemann

$$
\text { (4.) } \quad p=\frac{\omega}{2}-k+1 \text {. }
$$


$k$ ist nun gleich der Anzahl der Schnittlinien der Fläche mit einem linearen Complexe, also gleich dem Grade der Fläche; $\omega$ ist gleich der Anzahl der Werthe von $z$, für welche $\varphi^{\prime}(s)=0$ wird, ohne dass zugleich $\varphi^{\prime}(z)=0$ ist, d. h. gleich der Anzahl von Complexen des Büschels $\alpha+\beta z$, welche zwei benachharte Erzeugende mit der Fläche gemein haben oder sie berühren. Diese Zahl, welche man, nach der Analogie der Raumcurven, etwa den Rang der Fläche nennen könnte, und damit $p$, lässt sich bestimmen, wenn, wie wir jetzt annehmen wollen, die windschiefe Fläche der vollständige und nicht in Theile zerlegbare Schnitt der drei Complexe $u=0, v=0, w=0$ ist. Bezeichnen wir nämlich mit $a, b, c, d$ irgend vier Linien, so bestimmen diese ein Büschel von linearen Complexen und die Bedingung, dass einer derselben durch die Erzeugenden $x, x+d x$ gehe ist dann

$$
\Sigma \pm x_{1} d x_{2} a_{3} b_{4} c_{5} d_{6}=0 \text {. }
$$

Um diese Bedingung mit Hülfe der Gleichungen

$$
\begin{aligned}
& R=0, \quad v=0, \quad v=0, \quad w=0, \\
& \Sigma R_{i} d x_{i}=0, \quad \Sigma u_{i} d x_{i}=0, \quad \Sigma v_{i} d x_{i}=0, \quad \Sigma w_{i} d x_{i}=0, \quad\left(\varphi_{i}=\frac{\partial \varphi}{\partial x_{i}}\right)
\end{aligned}
$$

zu reduciren, multipliren wir mit

$$
\Sigma \pm u_{1} v_{2} w_{3} R_{4} \alpha_{5} \beta_{6}
$$

in welcher Determinante die $\alpha$ und $\beta$ beliebige Grössen sind, und erhalten dann, nach Fortlassung eines nicht verschwindenden Factors:

$$
\text { (5.) } \quad D=\left|\begin{array}{cccc}
\Sigma u_{i} a_{i} & \sum v_{i} a_{i} & \Sigma w_{i} a_{i} & \Sigma R_{i} a_{i} \\
\Sigma u_{i} b_{i} & \sum v_{i} b_{i} & \Sigma w_{i} b_{i} & \Sigma R_{i} b_{i} \\
\Sigma u_{i} c_{i} & \sum v_{i} c_{i} & \sum w_{i} c_{i} & \Sigma R_{i} c_{i} \\
\Sigma u_{i} d_{i} & \sum v_{i} d_{i} & \sum w_{i} d_{i} & \Sigma R_{i} d_{i}
\end{array}\right|=0 \text {. }
$$

Diese Gleichung ist vom Grade $l+m+n-2$ und der durch sie dargestellte Complex schneidet also die windschiefe Fläche in $2 \operatorname{lmn}(l+m+n-2)$ Linien. Dies ist die Zahl der gesuchten Complexe, vorausgesetzt, dass die Determinante, mit welcher multiplicirt wurde, nicht verschwindet. Dies geschieht aber unabhängig von den $\alpha$ und $\beta$, wenn entweder alle $u_{i} \operatorname{oder} v_{i}$ oder $w_{i}$ verschwinden, oder wenn eine Gleichung besteht von der Form

$$
\lambda_{1} u_{i}+\lambda_{2} v_{i}+\lambda_{3} w_{i}+\lambda_{4} R_{i}=0, \quad(i=1,2, \ldots 6)
$$

in welchen beiden Fällen die windschiefe Fläche Doppelerzeugende besitzt, für die mit $\varphi^{\prime}(s)$ auch $\varphi^{\prime}(z)$ zu Null wird. Ist die Anzahl dieser Linien $d$, 
so ist demnach

und somit, da hier $k=2 l m n$,

$$
\text { (6.) } \quad \omega=2 \operatorname{lmn}(l+m+n-2)-2 d \text {; }
$$

(7.) $p=\operatorname{lmn}(l+m+n-4)+1-d$.

Wenn die Fläche nicht der vollständige Schnitt dreier Complexe ist, ziehen wir aus der Gleichung (4.) das Resultat

$$
\text { (8.) } \quad \omega=2 k+2(p-1) \text {. }
$$

Um nun andere, auch für diesen Fall geltende, Formeln für $p$ zu erlangen, stellen wir die folgende Betrachtung an. Wie leicht zu sehen ist, sind die Coordinaten des Schnittpunktes einer Geraden und einer Ebene lineare Functionen der Coordinaten der Geraden. Schneiden wir also die windschiefe Fläche durch eine Ebene, so werden sich die Coordinaten eines Punktes der Schnittcurve darstellen als ganze Functionen von zwei Parametern, zwischen welchen eine Gleichung besteht, die auf $2 p$ fach periodische Functionen führt. Das Geschlecht der Schnittcurve ist also gleich dem Geschlechte der Fläche selbst* ${ }^{*}$ ). Die Schnittcurve hat einen Doppelpunkt, wo ihre Ebene eine Doppelcurve oder eine Doppelerzeugende schneidet. Bezeichnen wir also die Anzahl der Doppelerzeugenden mit $d$, die Summe der Grade der Doppelcurven mit $\delta$, so ist

$$
\text { (9.) } \quad p=\frac{k-1 . k-2}{2}-d-\delta \text {. }
$$

Andererseits stellen sich auch die Coordinaten einer Ebene, welche durch eine Gerade und einen Punkt geht, als lineare Functionen der Coordinaten der Geraden dar, und so findet man das Geschlecht der Fläche auch gleich dem Geschlechte des Tangentenkegels, den man von einem Punkte an sie legen kann, wenn man den Kegel als Einhüllende einer Schaar von Ebenen betrachtet. Die Klasse dieses Kegels ist aber gleich der Ordnung $k$ der Fläche; er hat eine Doppeltangentenebene, wo die Fläche eine Doppelerzeugende besitzt und ausserdem möge er noch $\delta^{\prime}$ Doppeltangentenebenen besitzen, welche Doppeltangentenebenen der Fläche sind. Dann ist

$$
p=\frac{k-1 \cdot k-2}{2}-d-\delta^{\prime}
$$

Wir sehen aus der Vergleichung beider Formeln für $p$ dass $\delta=\delta^{\prime}$, dass also die Summe der Ordnungen der Doppelcurven einer windschiefen Fläche gleich ist der Summe der Klassen der abwickelbaren Flächen der Doppeltangenten-

*) Dies wurde, soviel ich weiss, zuerst von Herrn H. Schwarz ausgesprochen (dieses Journal Bd. 64, pag. 2). 
ebenen. Wobei unter Klasse, wie gebräuchlich, die Anzahl der Ebenen verstanden ist, welche durch einen Punkt gehen. Mit Hülfe dieses Ausdrucks für $p$ finden wir noch

$$
\omega=k(k-1)-2 d-2 \delta .
$$

Wenn wir endlich aus (9.) $\delta$ berechnen und für $p$ seinen Werth (7.) anwenden, indem wir $k$ entprechend $=2 l m n$ setzen, so finden wir

$$
\delta=\operatorname{lmn}(2 \operatorname{lm} n-l-m-n+1)-d
$$

als die Summe der Ordnungen der Doppelcurven einer windschiefen Fläche, welche der vollständige Schnitt dreier Complexe ist.

§. 4. Die allenthalben endlichen Integrale.

Den im vorigen Paragraphen gefundenen Werth (7.) von $p$ kann man bestätigen durch directe Aufstellung der Integrale erster Gattung, deren es bekanntlich $p$ verschiedene giebt. Die Form dieser Integrale ist nach Herrn Clebsch (dieses Journal Bd. 63, pag. 224)

$$
\int \frac{\theta \Sigma \pm a_{1} b_{2} c_{3} d_{4} x_{5} d x_{6}}{D}
$$

wo $D$ die Bedeutung hat, die es im vorigen Paragraphen besass und wo $\theta$ eine homogene Function der Coordinaten ist vom Grade $l+m+n-4$. Diese Function enthält nun $(l+m+n+1)_{5}$ Glieder, von welchen sich jedoch mit Hülfe der gegebenen Gleichungen leicht manche zerstören lassen.

Es möge allgemein eine Function $k^{\text {ten }}$ Grades der sechs Grössen $x$ bestehen neben einer Gleichung von den Graden $k_{1}, k_{2}, k_{3}, k_{4}$, so sieht man leicht man, dass man, wenn $k>k_{1}+k_{2}+k_{3}+k_{4}$, mit Hülfe der gegebenen Gleichungen, in der Function $k^{\text {ten }}$ Grades so viele Glieder zerstören kann, dass nur noch

$$
(k+5)_{5}-\sum_{i}\left(k-k_{i}+5\right)_{5}+\sum_{i, x}\left(k-k_{i}-k_{x}+5\right)_{5}-\sum_{i, x, \lambda}\left(k-k_{i}-k_{x}-k_{\lambda}+5\right)_{5}+\left(k-k_{1}-k_{2}-k_{3}-k_{4}+5\right)_{5}
$$

übrig bleiben, wo $\sum_{i, \nu}$ die Summe bedeutet ausgedehnt über alle Combinationen

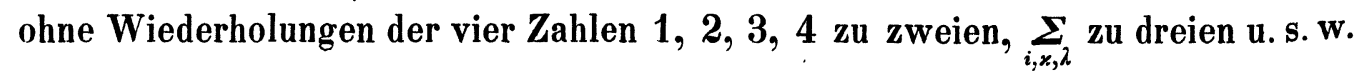
Um die obige Zahl auszuwerthen, beachten wir, dass $(n+5)_{5}$ der Coefficient ist von $x^{5}$ in der Entwickelung von $(1+x)^{n+5}$ nach Potenzen von $x$; der obige Ausdruck ist dann gleich dem Coefficienten von $x^{5}$ in der Entwickelung von

$$
\begin{gathered}
(1+x)^{k+5} \cdot\left\{1-(1+x)^{-k_{1}}\right\} \cdot\left\{1-\cdot(1+x)^{-k_{2}}\right\} \cdot\left\{1-(1+x)^{-k_{8}}\right\} \cdot\left\{1-(1+x)^{-k_{4}}\right\} \\
=x^{4} \cdot(1+x)^{k+5} \cdot k_{1} k_{2} k_{3} k_{4}\left\{1-\left(k_{1}+k_{2}+k_{3}+k_{4}+4\right) x+\cdots\right\}
\end{gathered}
$$


nach Potenzen von $x$, und findet sich also

$$
\text { (11.) }=k k_{1} k_{2} k_{3} k_{4}-\frac{k_{1} k_{2} k_{3} k_{4}}{2}\left(k_{1}+k_{2}+k_{3}+k_{4}-6\right) \text {. }
$$

Ist, wenn $k_{1}, k_{2}, k_{3}, k_{4}$ nach der Grösse geordnet sind, $k<k_{1}+k_{2}+k_{3}+k_{4}$ dagegen $>k_{2}+k_{3}+k_{4}$, so ist die obige Zahl zu vermindern um

$$
\left(k-k_{1}-k_{2}-k_{3}-k_{4}+5\right)_{5}
$$

d. h. zu vermehren um $\left(k_{1}+k_{2}+k_{3}+k_{4}-k-1\right)_{5}$. Wenn $k$ auch noch

$$
<k_{2}+k_{3}+k_{4} \text {, }
$$

so ist der Ausdruck weiter zu vermindern um $\left(k_{2}+k_{3}+k_{4}-k-1\right)_{5}$. Hier, wo $k=l+m+n-4$ und $k_{1}=2$, tritt der letzte Fall ein und es findet sich demnach die Zahl der Glieder

$$
\begin{gathered}
=2 l m n(l+m+n-4)-l m n(l+m+n-4)+1 \\
=l m n(l+m+n-4)+1 .
\end{gathered}
$$

Da aber der Complex $\theta$ noch durch die Doppelerzeugenden gehen muss, für welche die Determinante $D=0$, so ist diese Zahl noch zu vermindern um $d$, so dass in der That nur $p$ Glieder übrig bleiben, welche mit willkürlichen Constanten multiplicirt sind.

Die windschiefe Fläche wird von einem Complex in einer endlichen Anzahl Linien geschnitten. Wie nun Herr Clebsch a. a. O. gezeigt hat, ist die Summe von Abelschen Integralen erster Gattung ausgedehnt über dieses Schnittsystem constant, wenn der Complex durch die Doppelerzeugenden geht. Durch passende Wahl der unteren Grenzen, oder indem man von einem Schnittsystem bis zu einem anderen integrirt, kann dieser constante Werth gleich Null oder wenigstens gleich einem Periodicitätsmodul gemacht werden. Ist der Complex von der Ordnung $k$, so erhält man auf diese Weise zwischen den 2klmn-2d Schnittlinien beider Gebilde $p$ Gleichungen und $p$ dieser Linien sind also durch die übrigen bestimmt. Und in der That beträgt, wenn $k>$ $l+m+n-3$, die Anzahl der in $k$ noch bleibenden Constanten nach (11.)

$$
2 k l m n-\operatorname{lm} n(l+m+n-4) \text {. }
$$

Man kann also von dem Schnittsystem 2klmn-lmn $(l+m+n-4)-d-1$ Linien beliebig wählen und $\operatorname{lmn}(l+m+n-4)-d+1=p$ sind dadurch bestimmt. Die aus dem Abelschen Theorem fliessenden Gleichungen sind also gerade hinreichend zur Bestimmung dieser Erzeugenden. Wenn $k<l+m+n-3$, so wird die Zahl der durch die übrigen bestimmten Schnittlinien kleiner, ohne 
dass jedoch die Gleichungen des Abelschen Theorems zu bestehen aufhören können.

Man kann mit Hülfe des Abelschen Theorems, nach Analogie der von Herrn Clebsch bei den Curven aufgestellten Sätze, mit Leichtigkeit Sätze aufstellen über die Berührungen von Complexen mit windschiefen Flächen, die wir jedoch, da sie sich beinahe wörtlich aus jenen ablesen lassen, übergehen.

§. 5. Die singulären Erzeugenden.

Zwei aufeinanderfolgende Erzeugende einer windschiefen Fläche schneiden sich im Allgemeinen nicht, und es existirt nur eine endliche Anzahl von solchen, bei welchen es Statt hat. Wir wollen diese singuläre Erzeugende nennen und uns jetzt die Aufgabe stellen, deren Anzahl zu bestimmen, für den Fall einer windschiefen Fläche, welche der Schnitt dreier Complexe ist.

Es seien $x_{1}, \ldots x_{6}$ die Coordinaten einer Geraden, $x+d x$ die einer benachbarten. Es bestehen dann die Gleichungen

$$
\boldsymbol{R}(\boldsymbol{x})=0, \quad R(x+d x)=\Sigma d x_{i} \frac{\partial R}{\partial x_{i}}+\frac{1}{2} \Sigma d x_{i} d x_{k} \frac{\partial^{2} R}{\partial x_{i} \partial x_{k}}=0 .
$$

Sollen sich beide Linien schneiden, so muss

$$
\Sigma\left(x_{i}+d x_{i}\right) \frac{\partial R}{\partial x_{i}}=0
$$

sein. Verbindet man diese Gleichung mit der vorigen, so zeigt sich, dass, wenn zwei Systeme von Grössen $x, x+d x$ die Coordinaten zweier sich schneidenden Linien sein sollen, die Gleichungen bestehen müssen

$$
\text { (12.) } \quad R(x)=0, \quad \sum R_{i} d x_{i}=0, \quad \sum \frac{\partial^{2} R}{\partial x_{i} \partial x_{k}} d x_{i} d x_{k}=0 .
$$

Neben diesen gelten für unseren Fall die Gleichungen

$$
\begin{array}{ccc}
u=0, & v=0, & w=0, \\
\Sigma u_{i} d x_{i}=0, & \Sigma v_{i} d x_{i}=0, & \Sigma w_{i} d x_{i}=0 .
\end{array}
$$

Bestimmen wir aus den drei letzten Gleichungen, der zweiten (12.) und einer Gleichung

$$
\Sigma k_{i} d x_{i}=0,
$$

welche aus einer Beziehung $\Sigma k_{i} x_{i}=1$ zwischen den homogenen Coordinaten hervorgeht, die Verhältnisse der $d x$ und setzen sie in die letzte Gleichung (12.) ein, so erhalten wir in der mit 


$\begin{array}{llllll}u_{1} & u_{2} & u_{3} & u_{4} & u_{5} & u_{6} \\ v_{1} & v_{2} & v_{3} & v_{4} & v_{5} & v_{6} \\ w_{1} & w_{2} & w_{3} & w_{4} & w_{5} & w_{6} \\ \boldsymbol{R}_{1} & \boldsymbol{R}_{2} & \boldsymbol{R}_{3} & \boldsymbol{R}_{4} & \boldsymbol{R}_{5} & \boldsymbol{R}_{6} \\ \boldsymbol{k}_{1} & \boldsymbol{k}_{2} & \boldsymbol{k}_{3} & \boldsymbol{k}_{4} & \boldsymbol{k}_{5} & \boldsymbol{k}_{6}\end{array}$

geränderten Hesseschen Determinante von $\boldsymbol{R}$ die Gleichung des Complexes, welcher die windschiefe Fläche in den gesuchten Erzeugenden schneidet. In der so erhaltenen Gleichung kommen die willkürlichen Constanten $k$ noch im zweiten Grade vor. Diese kann man auf bekannte Weise in einen Factor $\left(k_{1} x_{1}+\cdots+k_{6} x_{6}\right)^{2}=1$ vereinigen und erlangt so endlich die Gleichung des Complexes, wenn man noch die Werthe der $\frac{\partial^{2} R}{\partial x_{i} \partial x_{k}}$ einträgt:

$$
\left|\begin{array}{lllllllll}
0 & 0 & 0 & 1 & 0 & 0 & u_{1} & v_{1} & w_{1} \\
0 & 0 & 0 & 0 & 1 & 0 & u_{2} & v_{2} & w_{2} \\
0 & 0 & 0 & 0 & 0 & 1 & u_{3} & v_{3} & w_{3} \\
1 & 0 & 0 & 0 & 0 & 0 & u_{4} & v_{4} & w_{4} \\
0 & 1 & 0 & 0 & 0 & 0 & u_{5} & v_{5} & w_{5} \\
0 & 0 & 1 & 0 & 0 & 0 & u_{6} & v_{6} & w_{6} \\
u_{1} & u_{2} & u_{3} & u_{4} & u_{5} & u_{6} & 0 & 0 & 0 \\
v_{1} & v_{2} & v_{3} & v_{4} & v_{5} & v_{6} & 0 & 0 & 0 \\
w_{1} & w_{2} & w_{3} & w_{4} & w_{5} & w_{6} & 0 & 0 & 0
\end{array}\right|=0 .
$$

Dieser Complex ist vom Grade $2(l+m+n-3)$. Die Anzahl seiner Schnittlinien mit der Fläche ist somit $=4 \operatorname{lmn}(l+m+n-3)$. Jede Doppelerzeugende ist aber auch Doppelerzeugende dieses Complexes, und somit sind von dieser Zahl die $4 d$ Schnittlinien, welche in Doppelerzeugende fallen, fortzulassen, weil sich dort wohl benachbarte, aber nicht aufeinanderfolgende Erzengende schneiden. Es bleibt also die Zahl der singulären Erzeugenden

$$
\text { (13.) }\left\{\begin{array}{l}
=4 l m n(l+m+n-3)-4 d \\
=2 k+4(p-1) .
\end{array}\right.
$$

Für ein Hyperboloid ist $k=2, p=0$. Daher die Zahl wie bekannt $=0$. Setzen wir $l=2, m=1, n=1$, so wird $k=4, p=1$ und also die Zahl der singulären Erzeugenden $=8$. 
§. 6. Beziehung der windschiefen Fläche auf eine Curve.

Wir haben gesehen, dass die Coordinaten der Erzeugenden einer windschiefen Fläche sich darstellen als homogene Functionen dreier Parameter, zwischen welchen eine Gleichung besteht. Fassen wir diese Parameter als die Dreieckscoordinaten eines Punktes in einer Ebene auf, so stellt die Gleichung zwischen ihnen eine Curve dar und es ist also, durch die homogenen Functionen, eine solche Beziehung festgesetzt zwischen der Fläche und der Curve, dass, im Allgemeinen, jedem Punkte der Curve eine Erzeugende der Fläche entspricht und umgekehrt. Jedem Punkte der Ebene wird aber auch eine gerade Linie zugehören, deren Coordinaten man findet, indem man in jene Functionen die Coordinaten des Punktes einträgt; alle diese Linien bilden eine zweifach unendliche Schaar, d. h. ein Strahlensystem und sind durch gewisse Eigenthümlichkeiten an einander geknüpft. Jeder Curve der Ebene entspricht dann eine windschiefe Fläche des Strahlensystems, und der Geometrie der Ebene eine Geometrie im Strahlensystem.

Es mögen die Coordinaten $x$ einer geraden Linie gegeben sein als homogene Functionen $m^{\text {ter }}$ Ordnung

$$
\text { (14.) } \quad \varrho x_{i}=\varphi^{(i)}\left(y_{1} y_{2} y_{3}\right) \text {, }
$$

wo $\varrho$ ein Proportionalitätsfactor ist, und die Functionen $\varphi$ als so beschaffen vorausgesetzt werden, dass sie die Gleichung

$$
\varphi^{(1)} \varphi^{(4)}+\varphi^{(2)} \varphi^{(5)}+\varphi^{(3)} \varphi^{(6)}=0
$$

identisch erfüllen.

Wir wollen ferner voraussetzen, dass Punkte, Ausnahmspunkte, vorhanden sind, für welche alle Functionen $\varphi$ gleichzeitig verschwinden; und zwar mögen die Curven $\varphi=0 \mathrm{im} i^{\text {ten }}$ Ausnahmspunkte alle einen $\eta_{i}$ fachen Punkt haben. Da für einen solchen Punkt die linken Seiten von (14.) gleich Null werden, so müssen entweder alle $x$ verschwinden, was keinen Sinn hat, oder $\varrho$ muss Null sein und zwar $\eta$ fach. Differentiiren wir nun die Gleichungen (14.) $\eta$ mal, so erhalten wir, da für den Ausnahmspunkt $d \varrho=d^{2} \varrho=\cdots=d^{\eta-1} \varrho=0$,

$$
d^{\eta} \varphi \cdot x_{i}=\Sigma d y_{1}^{\alpha_{1}} d y_{2}^{\alpha_{2}} d y_{3}^{\alpha_{3}} \cdot \partial_{\alpha}^{\eta} \varphi^{(i)}
$$

wo der Kürze wegen $\partial_{\alpha}^{\eta} \varphi^{(i)}$ für $\frac{\partial^{\eta} \varphi^{(i)}}{\partial y_{1}^{\alpha_{1}} \partial y_{2}^{\alpha_{2}} \partial y_{3}^{\alpha_{3}}}$ gesetzt ist. Man sieht hieraus, dass jeder Richtung in der Ebene der $y$, in der man vom Ausnahmspunkte fortgeht, eine bestimmte Linie entspricht. 
Denken wir uns nun in der Ebene der. $y$ eine Curve $n^{\text {ter }}$ Ordnung $f=0$, welche im $i^{\text {ten }}$ Ausnahmspunkte einen $\alpha_{i}$ fachen Punkt hat, und bestimmen die Ordnung der ihr entsprechenden Fläche. Diese ist gleich der Anzahl von Schnittpunkten mit einer Geraden $a$ oder gleich der Anzahl der gemeinsamen Wurzeln der Gleichungen

$$
f=0, \quad \Sigma \varphi^{(i)} \frac{\partial R}{\partial a_{i}}=0 .
$$

Es sind deren m.n. Da aber die zweite Gleichung, unabhängig von den $a$ befriedigt wird durch die Coordinaten der Ausnahmspunkte, so sind die diesen entsprechenden Schnittpunkte zu verwerfen und der Grad der Fläche ist also

$$
\text { (16.) } k=m \cdot n-\Sigma \alpha_{i} \eta_{i} \text {. }
$$

Das Geschlecht der Fläche ist gleich dem Geschlechte der Curve $f$ und somit, wenn wir mit $\beta$ die Anzahl der Zweige eines vielfachen Punktes bezeichnen, der kein Ausnahmspunkt ist

$$
\text { (17.) } \quad p=\frac{n-1 . n-2}{2}-\Sigma \frac{\alpha_{i}\left(\alpha_{i}-1\right)}{2}-\Sigma \frac{\beta_{i}\left(\beta_{i}-1\right)}{2} \text {. }
$$

Die Fläche wird dann die Erzeugenden, die vielfachen Punkten entsprechen, welche nicht zugleich Ausnahmspunkte sind, zu vielfachen Erzeugenden haben, und zwar zu $\boldsymbol{\beta}$ fachen, wenn der betreffende Punkt $\boldsymbol{\beta}$ Zweige hat. Ausserdem werden noch Doppelerzeugende auftreten, welche von der Natur des Strahlensystems herrühren. Deren Anzahl plus der Summe der Grade der Doppelcurven findet man aus (9.) $\$$. 3, indem man die oben gefundenen Werthe für $k$ und $p$ einträgt und von der berechneten Summe $d+\delta$ noch $\sum \frac{\beta_{i}\left(\beta_{i}-1\right)}{2}$ abzieht.

Es ist hier, wie im Folgenden, vorausgesetzt, dass die vielfachen Punkte keine zusammenfallenden Tangenten haben.

§. 7. Der Rang der windschiefen Fläche.

Die Zahl der linearen Complexe eines Büschels, welche eine windschiefe Fläche berühren, könnten wir, mit Hülfe der obigen Zahlen, aus (8.) berechnen. Wir wollen jedoch hier, um eine Anwendung der eben entwickelten Principien zu machen, jene Formel direct verificiren. 
Die Gleichung des Büschels sei $u+\lambda v=0$, wo $u$ und $v$ lineare Ausdrücke in den $x$ sind. Wenn man nun in der Gleichung eines Complexes für die Coordinaten $x$ die $\varphi$ setzt, so erhält man die Gleichung einer Curve, deren Schnittpunkte mit $f=0$ den Schnittlinien des Complexes mit der Fläche entsprechen. Aus dem Büschel von Complexen $u+\lambda v=0$ entsteht so ein Curvenbüschel und der gesuchte Rang der Fläche ist gleich der Anzahl von Curven dieses Büschels, welche $f=0$ berühren, d. h. gleich der Anzahl der Schnittpunkte dieser Curve mit der Jacobischen Curve der Functionen $f, u, v$, deren Gleichung $\boldsymbol{T}=\mathbf{0}$ wir abkürzend schreiben wollen

$$
\text { (18.) } T=\left|\begin{array}{c}
f_{i} \\
u_{i} \\
v_{i}
\end{array}\right|=0 \text {. }
$$

Diese Curve ist von der Ordnung $2 m+n-3$ und die Anzahl ihrer Schnittlinien mit $f=0$, also $n(2 m+n-3)$. Hievon gehen aber einige ab. Hat $f=0$ einen $\beta$ fachen Punkt, der kein Ausnahmspunkt ist, so hat $T$ einen $(\beta-1)$ fachen und die hier entstehenden $\beta(\beta-1)$ Punkte sind nicht zu zählen.

Hat aber $f$ einen $\alpha$ fachen Punkt in einem $\eta$ fachen Ausnahmspunkt, so wird $T(2 \eta+\alpha-3)$ fach Null. Um die Natur dieses Punktes näher zu untersuchen, setzen wir in $T$ für $y y+\lambda z$, wo unter $y$ die Coordinaten des betrachteten Punktes verstanden sind und entwickeln. Das erste Glied, welches nicht verschwindet, enthält $\dot{\lambda}^{2 \eta+\alpha-3}$, dessen Coefficient ist

$$
\left|\begin{array}{l}
\partial^{\alpha-1} f_{i} \\
\partial^{\eta-1} u_{i} \\
\partial^{\eta-1} v_{i}
\end{array}\right|=\partial^{2 \eta+\alpha-3} T
$$

wo $\partial^{\eta} \varphi$ symbolisch gleich ist $\frac{1}{\eta !} \cdot\left(z_{1} \frac{\partial}{\partial y_{1}}+z_{2} \frac{\partial}{\partial y_{2}}+z_{3} \frac{\partial}{\partial y_{3}}\right)^{\eta} \varphi$. Aber auch dieser Coefficient ist gleich Null. Um dies zu zeigen, bilden wir, unter $A$ beliebige Grössen verstanden,

$$
\left(A_{1} y_{1}+A_{2} y_{2}+A_{3} y_{3}\right) \partial^{2 \eta+\kappa-3} T=\mid \begin{gathered}
A_{1} y_{1}+A_{2} y_{2}+A_{3} y_{3} \\
0 \\
0 \\
0
\end{gathered}
$$$$
\begin{gathered}
A_{i} \\
\partial^{\alpha-1} f_{i} \\
\partial^{\eta-1} u_{i} \\
\partial^{\eta-1} v_{i}
\end{gathered} \mid
$$

Aus der symbolischen Darstellung von $\partial^{\eta} \varphi$ folgen nun leicht die Gleichungen

$$
\partial^{\eta-1} \varphi_{i}=\frac{\partial}{\partial y_{i}} \partial^{\eta-1} \varphi=\frac{\partial}{\partial z_{i}} \partial^{\eta} \varphi
$$


mit deren Hülfe die rechte Seite der vorigen Gleichung

$$
=-\left|\begin{array}{cc}
0 & A_{i} \\
(n-\alpha+1) \partial^{\alpha-1} f & \partial^{\alpha-1} f_{i} \\
(m-\eta+1) \partial^{\eta-1} u & \partial^{\eta-1} u_{i} \\
(m-\eta+1) \partial^{\eta-1} v & \partial^{\eta-1} v_{i}
\end{array}\right|
$$

wird. Dieser Ausdruck verschwindet aber, da ja $\partial^{\alpha-1} f$ und $\partial^{\eta-1} u, \partial^{\eta-1} v$ verschwinden, weil $f$ einen $\alpha$ fachen, $u$ und $v$ aber einen $\eta$ fachen Punkt haben. Wir müssen also den Coefficienten von $\lambda^{2 \eta+\alpha-2}$ betrachten, der sich findet

$$
\partial^{2 \eta+\alpha-2} \boldsymbol{T}=\left|\begin{array}{c}
\partial^{\alpha} f_{i} \\
\partial^{\eta-1} u_{i} \\
\partial^{\eta-1} v_{i}
\end{array}\right|+\left|\begin{array}{c}
\partial^{\alpha-1} f_{i} \\
\partial^{\eta} u_{i} \\
\partial^{\eta-1} v_{i}
\end{array}\right|+\left|\begin{array}{c}
\partial^{\alpha-1} f_{i} \\
\partial^{\eta-1} u_{i} \\
\partial^{\eta} v_{i}
\end{array}\right| .
$$

Multipliciren wir wieder mit $A_{1} y_{1}+A_{2} y_{2}+A_{3} y_{3}$, so geht mit Benutzung der früheren Gleichungen der erste Term über in

$$
-\left|\begin{array}{cc}
0 & A_{i} \\
(n-\alpha) \partial^{\alpha} f & \partial^{\alpha} f_{i} \\
0 & \partial^{\eta-1} u_{i} \\
0 & \partial^{\eta-1} v_{i}
\end{array}\right|
$$

und ähnlich verwandeln sich der zweite und dritte Term, so dass man schreiben kann

$$
\left(A_{1} y_{1}+A_{2} y_{2}+A_{3} y_{3}\right) \partial^{2 \eta+\alpha-2} T=-\left|\begin{array}{cc}
0 & A_{i} \\
(n-\alpha) \partial^{\alpha} f & \partial^{\alpha-1} f_{i} \\
(m-\eta) \partial^{\eta} u & \partial^{\eta-1} u_{i} \\
(m-\eta) \partial^{\eta} v & \partial^{\eta-1} v_{i}
\end{array}\right|
$$

Multiplicirt man die drei letzten Reihen mit $\frac{m-\eta}{\eta} z_{1}, \frac{m-\eta}{\eta} z_{2}, \frac{m-\eta}{\eta} z_{3}$ und subtrahirt von der ersten, so kommt:

(19.)

$$
\left\{\begin{array}{c}
\left(A_{1} y_{1}+A_{2} y_{2}+A_{3} y_{3}\right) \partial^{2 \eta+\alpha-2} T=-\left|\begin{array}{cc}
\frac{m-\eta}{\eta}\left(A_{1} \eta_{1}+A_{2} \eta_{2}+A_{3} \eta_{3}\right) & A_{i} \\
\frac{n \eta-m \alpha}{\eta} \partial^{\alpha} f & \partial^{\alpha-1} f_{i} \\
0 & \partial^{\eta-1} u_{i} \\
0 & \partial^{\eta-1} v_{i}
\end{array}\right| \\
=\frac{n \eta-m \alpha}{\eta} \partial^{\alpha} f \cdot\left|\begin{array}{c}
\partial A_{i} \\
\partial^{\eta-1} u_{i} \\
\partial^{\eta-1} v_{i}
\end{array}\right| .
\end{array}\right.
$$


Die Curve $T$ hat also einen $(2 \eta+\alpha-2)$ fachen Punkt, von dessen Tangenten $\alpha$ mit denen des Punktes von $f=0$ zusammenfallen; und die Anzahl der fortzulassenden Schnittpunkte ist somit $\alpha(\alpha+1)+(2 \eta-2) \alpha$. Die gesuchte Zahl wird also :

$$
\begin{aligned}
& =n(2 m+n-3)-\Sigma \beta_{i}\left(\beta_{i}-1\right)-\Sigma 2 \eta_{i} \alpha_{i}-\Sigma \alpha_{i}\left(\alpha_{i}-1\right) \\
& =2 k+2(p-1),
\end{aligned}
$$

wie wir oben gefunden hatten.

§. 8. Die singulären Erzeugenden.

Wir wollen jetzt die Anzahl der singulären Erzeugenden bestimmen, welche auf der durch die Curve $f=0$ dargestellten Fläche liegen.

Die Bedingung, dass sich zwei durch die Punkte $y$ und $y+d y$ repräsentirte Linien schneiden, ist die

$$
\begin{gathered}
0=\varphi^{(1)}(y) \varphi^{(4)}(y+d y)+\varphi^{(4)}(y) \varphi^{(1)}(y+d y)+\varphi^{(2)}(y) \varphi^{(5)}(y+d y)+\varphi^{(5)}(y) \varphi^{(2)}(y+d y) \\
+\varphi^{(3)}(y) \varphi^{(6)}(y+d y)+\varphi^{(6)}(y) \varphi^{(3)}(y+d y) .
\end{gathered}
$$

Entwickeln wir hier die $\varphi(y+d y)$ und bedenken, dass der identischen Gleichung (15.) wegen,

$$
\left\{\begin{array}{l}
\varphi^{(1)} \varphi_{i}^{(4)}+\varphi^{(4)} \varphi_{i}^{(1)}+\varphi^{(2)} \varphi_{i}^{(5)}+\varphi^{(5)} \varphi_{i}^{(2)}+\varphi^{(3)} \varphi_{i}^{(6)}+\varphi^{(6)} \varphi_{i}^{(3)}=0 \\
\varphi^{(1)} \varphi_{i k}^{(4)}+\varphi^{(4)} \varphi_{i k}^{(1)}+\cdots+\varphi_{i}^{(1)} \varphi_{k}^{(4)}+\varphi_{i}^{(4)} \varphi_{k}^{(1)}+\cdots=0
\end{array}\right.
$$

so verschwinden die Glieder erster Ordnung und die Glieder zweiter Ordnung schreiben sich,

$$
\varphi_{i}^{(1)} \varphi_{k}^{(4)}+\varphi_{k}^{(1)} \varphi_{i}^{(4)}+\varphi_{i}^{(2)} \varphi_{k}^{(5)}+\varphi_{k}^{(2)} \varphi_{i}^{(5)}+\varphi_{i}^{(3)} \varphi_{k}^{(6)}+\varphi_{k}^{(3)} \varphi_{i}^{(6)}=a_{i k}
$$

gesetzt,

$$
\text { (22.) } \quad \sum_{i, k} a_{i k} d y_{i} d y_{k}=0 \text {. }
$$

Setzen wir hier für die Verhältnisse der $d y$ die Werthe, welche sich aus den Gleichungen

$$
\Sigma f_{i} d y_{i}=0, \quad \Sigma k_{i} d y_{i}=0
$$

ergeben, so erhalten wir in

$$
\text { (23.) } . S=\left|\begin{array}{lllll}
a_{11} & a_{12} & a_{13} & f_{1} & k_{1} \\
a_{21} & a_{22} & a_{23} & f_{2} & k_{2} \\
a_{31} & a_{32} & a_{33} & f_{3} & k_{3} \\
f_{1} & f_{2} & f_{3} & 0 & 0 \\
k_{1} & k_{2} & k_{3} & 0 & 0
\end{array}\right|=0
$$


die Gleichung der Curve, welche $f=0$ in den Punkten schneidet, welchen singuläre Erzeugende entsprechen. Diese Gleichung enthält noch die Grössen $k$ im zweiten Grade, und diese müssen in einem Factor abgesondert werden. Dass dies möglich ist, sieht man leicht ein. Denn bildet man, einen Ausdruck wie $a_{1} y_{1}+a_{2} y_{2}+a_{3} y_{3}=a$ gesetzt, $S . l^{2}$, indem man der obigen Determinante noch die Zeilen

$$
\begin{array}{lllllll}
l_{1} & l_{2} & l_{3} & 0 & 0 & 0 & l \\
0 & 0 & 0 & 0 & 0 & l & 0
\end{array}
$$

und die entsprechenden Colonnen zufügt, so kann man diese Gleichung leicht transformiren. Es dienen hierzu die aus (21.) wegen des Satzes der homogenen Functionen hervorgehenden identischen Gleichungen

$$
\text { (24.) } \quad \sum_{i} a_{i k} y_{i}=0, \quad(k=1,2,3) \text {. }
$$

Die transformirte Determinante ist dann gleich $S^{\prime} . k^{2}$, wo $S^{\prime}$ sich von $S$ nur dadurch unterscheidet, dass die $l$ an die Stelle der $k$ getreten sind. Aus der Gleichung $S . l^{2}=S^{\prime} . k^{2}$ folgt aber, dass $\frac{S}{k^{2}}$ von den $k$ frei sein muss, w. z. b. w. Um den Factor $k^{2}=1$ wirklich abzusondern, bedarf es einer genaueren Untersuchung der Form der Grössen $a_{i k}$. Wir glauben diese hier um so mehr übergehen zu können, als die Untersuchung der Singularitäten von $S$ sich leichter an die obige Form anschliesst, und die andere, von $k^{2}$ befreite Form, keine Vortheile bietet.

Die Curve $S=0$ ist von der Ordnung $2(m+n-3)$ und hat also $2 n(m+n-3)$ Schnittpunkte mit $f=0$. Von diesen Punkten sind jedoch manche zu verwerfen. Hat zunächst die Curve $f=0$ einen $\beta$ fachen Punkt, der kein Ausnahmspunkt ist, so hat $S$ dort einen $(2 / \beta-2)$ fachen Punkt, und die Anzahl der in diesem Punkte vereinigten Schnittpunkte beträgt somit $2 \beta(\beta-1)$. Diese sind aber nicht zu zählen, weil sich in der entsprechenden Linie wohl unendlich nahe aber nicht aufeinanderfolgende Erzeugende schneiden.

Hat aber die Curve $f$ einen $\alpha$ fachen Punkt in einem Ausnahmspunkt, so ist der Fall, dass dieser ein einfacher Punkt aller Curven $\varphi=0$ ist, von dem zu trennen, dass er ein vielfacher Punkt ist.

Im ersten der beiden Fälle wird $S(2 \alpha-1)$ fach Null, da, wie aus (20.) erhellt, $a_{i k}$ daselbst Null wird. Um die Natur dieses Punktes zu erforschen, haben wir $\mathrm{zu}$ betrachten 


$$
\partial^{2 \alpha-1} S=\left|\begin{array}{lllll}
\partial a_{11} & \partial a_{12} & \partial a_{13} & \partial^{\alpha-1} f_{1} & k_{1} \\
\partial a_{21} & \partial a_{22} & \partial a_{23} & \partial^{\alpha-1} f_{2} & k_{2} \\
\partial a_{31} & \partial a_{32} & \partial a_{33} & \partial^{\alpha-1} f_{3} & k_{3} \\
\partial^{\alpha-1} f_{1} & \partial^{\alpha-1} f_{2} & \partial^{\alpha-1} f_{3} & 0 & 0 \\
k_{1} & k_{2} & k_{3} & 0 & 0
\end{array}\right| .
$$

Mit Hülfe der Gleichungen, welche die $a_{i k}$ definiren, erkennen wir nun leicht, dass für einen Ausnahmspunkt die Gleichung Statt findet:

$$
\frac{\partial a_{i k}}{\partial y_{h}}+\frac{\partial a_{h k}}{\partial y_{i}}+\frac{\partial a_{h i}}{\partial y_{k}}=0
$$

aus der sich die speciellen Fälle

ergeben.

$$
\frac{\partial a_{h h}}{\partial y_{h}}=\mathbf{0} ; \quad \frac{\partial a_{i k}}{\partial y_{i}}=-\frac{1}{2} \frac{\partial a_{i i}}{\partial y_{k}}
$$

Weil ferner für einen Ausnahmspunkt $a_{i k}=0$, so hat man auch die Gleichung:

$$
0=\frac{\partial a_{i k}}{\partial y_{1}} y_{1}+\frac{\partial a_{i k}}{\partial y_{2}} y_{2}+\frac{\partial a_{i k}}{\partial y_{3}} y_{3} \text {. }
$$

Berechnet man aus diesen Formeln die Werthe der Differentialquotienten, so findet man schliesslich

$$
\begin{array}{ll}
\partial a_{11}=2 M\left(y_{3} z_{2}-y_{2} z_{3}\right), & \partial a_{12}=-M y_{3} z_{1}+N y_{3} z_{2}+\left(M y_{1}-N y_{2}\right) z_{3}, \\
\partial a_{22}=2 N\left(y_{1} z_{3}-y_{3} z_{1}\right), & \partial a_{23}=\left(N y_{2}-P y_{3}\right) z_{1}-N y_{1} z_{2}+P y_{1} z_{3}, \\
\partial a_{33}=2 P\left(y_{2} z_{1}-y_{1} z_{2}\right), & \partial a_{31}=M y_{2} z_{1}+\left(P y_{3}-M y_{1}\right) z_{2}-P y_{2} z_{3} .
\end{array}
$$

Aus der identischen Gleichung $\sum_{i} a_{i k} y_{i}=0$ folgt noch die Gleichung $\Sigma \partial a_{i k} y_{i}=0$, deren Bestehen erfordert, dass

$$
M y_{1}+N y_{2}+P y_{3}=0
$$

sei. Dieser Gleichung genügend kann man setzen

$$
M: N: P=A_{2} y_{3}-A_{3} y_{2}: A_{3} y_{1}-A_{1} y_{3}: A_{1} y_{2}-A_{2} y_{1} \text {. }
$$

Führt man nun die Werthe der $\partial a_{i k}$ in $\partial^{2 \alpha-1} S$ ein, so findet sich dies

$$
=\left|\begin{array}{lll}
y_{1} & z_{1} & k_{2} \partial^{\alpha-1} f_{3}-k_{3} \partial^{\alpha-1} f_{2} \\
y_{2} & z_{2} & k_{3} \partial^{\alpha-1} f_{1}-k_{1} \partial^{\alpha-1} f_{3} \\
y_{3} & z_{3} & k_{1} \partial^{\alpha-1} f_{2}-k_{2} \partial^{\alpha-1} f_{1}
\end{array}\right| \cdot\left|\begin{array}{ccc}
k_{1} & \partial^{\alpha-1} f_{1} & A_{2} y_{3}-A_{3} y_{2} \\
k_{2} & \partial^{\alpha-1} f_{2} & A_{3} y_{1}-A_{1} y_{3} \\
k_{3} & \partial^{\alpha-1} f_{3} & A_{1} y_{2}-A_{2} y_{1}
\end{array}\right| .
$$

Reducirt man diese beiden Determinanten auf die bekannte Art, so findet sich endlich

$$
\partial^{2 \alpha-1} S=\partial^{\alpha} f \cdot\left(A_{1} \partial^{\alpha-1} f_{1}+A_{2} \partial^{\alpha-1} f_{2}+A_{3} \partial^{\alpha-1} f_{3}\right) \cdot k^{2}
$$


Die Curve $S$ hat also im Ausnahmspunkt einen $(2 \alpha-1)$ fachen Punkt, von dessen Tangenten $\alpha$ mit denen des Punktes von $f$ coincidiren. Dieser Punkt absorbirt somit $\alpha(\alpha+1)+\alpha(\alpha-1)=2 \alpha^{2}$ Schnittpunkte, die nicht zu zählen sind.

Wir betrachten jetzt den Fall. dass der Ausnahmspunkt ein $\eta$ facher Punkt ist, wo $\eta \geqq 2$. Da, wie man aus (21.) sieht, die $a_{i k}(2 \eta-2)$ fach Null werden, so hat $S$ einen $(2 \eta+2 \alpha-4)$ fachen Punkt.

Um ihn genauer zu untersuchen, betrachten wir die Polare

$$
\partial^{2 \eta+2 \alpha-4} S=\left|\begin{array}{lllll}
\partial^{2 \eta-2} a_{11} & \partial^{2 \eta-2} a_{12} & \partial^{2 \eta-2} a_{13} & \partial^{\alpha-1} f_{1} & k_{1} \\
\partial^{2 \eta-2} a_{21} & \partial^{2 \eta-2} a_{22} & \partial^{2 \eta-2} a_{23} & \partial^{\alpha-1} f_{2} & k_{2} \\
\partial^{2 \eta-2} a_{31} & \partial^{2 \eta-2} a_{32} & \partial^{2 \eta-2} a_{33} & \partial^{\alpha-1} f_{3} & k_{3} \\
\partial^{\alpha-1} f_{1} & \partial^{\alpha-1} f_{2} & \partial^{\alpha-1} f_{3} & 0 & 0 \\
k_{1} & k_{2} & k_{3} & 0 & 0
\end{array}\right| .
$$

Da nun die Gleichung

$$
a_{i 1} y_{1}+a_{i 2} y_{2}+\dot{a}_{i 3} y_{3}=0 \quad(i=1,2,3)
$$

eine identische ist, so gelten für den Ausnahmspunkt die Gleichungen

$$
\text { (26.) } \quad y_{1} \partial^{2 \eta-2} a_{i 1}+y_{2} \partial^{2 \eta-2} a_{i 2}+y_{3} \partial^{2 \eta-2} a_{i 3}=0 \text {. }
$$

Aus der Gleichung

$$
\partial^{2 \eta-2} a_{i k}=\partial^{\eta-1} \varphi_{i}^{(1)} \partial^{\eta-1} \varphi_{k}^{(4)}+\partial^{\eta-1} \varphi_{k}^{(1)} \partial^{\eta-1} \varphi_{i}^{(4)}+\cdots,
$$

die, wegen

$$
\partial^{\eta-1} \varphi_{i}=\frac{\partial}{\partial z_{i}} \partial^{\eta} \varphi
$$

auch geschrieben werden kann:

$$
\partial^{2 \eta-2} a_{i k}=\frac{\partial}{\partial z_{i}} \partial^{\eta} \varphi^{(1)} \cdot \frac{\partial}{\partial z_{k}} \partial^{\eta} \varphi^{(4)}+\frac{\partial}{\partial z_{i}} \partial^{\eta} \varphi^{(4)} \cdot \frac{\partial}{\partial z_{k}} \partial^{\eta} \varphi^{(1)}+\cdots,
$$

folgt nun

$$
\Sigma z_{i} \partial^{2 \eta-2} a_{i k}=\partial^{\eta} \varphi^{(1)} \frac{\partial}{\partial z_{k}} \partial^{\eta} \varphi^{(4)}+\partial^{\eta} \varphi^{(4)} \cdot \frac{\partial}{\partial z_{k}} \partial^{\eta} \varphi^{(1)}+\cdots
$$

Die rechte Seite dieser Gleichung ist identisch Null, wie sich sogleich zeigt, wenn man die Operation $\partial^{2 \eta}$ auf die identische Gleichung

$$
\varphi^{(1)} \varphi^{(4)}+\varphi^{(2)} \varphi^{(5)}+\varphi^{(3)} \varphi^{(6)}=0
$$

anwendet, für die $y$ die Coordinaten des Ausnahmspunktes setzt und dann nach $z_{k}$ differentiirt. Wir haben also auch die Gleichungen

$$
z_{1} \partial^{2 \eta-2} a_{i 1}+z_{2} \partial^{2 \eta-2} a_{i 2}+z_{3} \partial^{2 \eta-2} a_{i 3}=0 \quad(i=1,2,3) .
$$


Wenn wir eine der Gleichungen (26.) mit der entsprechenden Gleichung (27.) verbinden, so können wir die Verhältnisse von

$$
\partial^{2 \eta-2} a_{i 1}, \quad \partial^{2 \eta-2} a_{i 2}, \quad \partial^{2 \eta-2} a_{i 3}
$$

berechnen.' Durch Vergleich der beiden Werthe von $\partial^{2 \eta-2} a_{12}, \partial^{2 \eta-2} a_{23}, \partial^{2 \eta-2} a_{31}$ können wir die auftretenden Proportionalitätsfactoren bis auf einen bei allen Grössen gleichen Factor bestimmen und finden so endlich:

$$
\begin{array}{ll}
\partial^{2 \eta-2} a_{11}=A\left(y_{2} z_{3}-y_{3} z_{2}\right)^{2}, & \partial^{2 \eta-2} a_{12}=A\left(y_{2} z_{3}-y_{3} z_{2}\right)\left(y_{3} z_{1}-y_{1} z_{3}\right), \\
\partial^{2 \eta-2} a_{22}=A\left(y_{3} z_{1}-y_{1} z_{3}\right)^{2}, & \partial^{2 \eta-2} a_{23}=A\left(y_{3} z_{1}-y_{1} z_{3}\right)\left(y_{1} z_{2}-y_{2} z_{1}\right), \\
\partial^{2 \eta-3} a_{33}=A\left(y_{1} z_{2}-y_{2} z_{1}\right)^{2}, & \partial^{2 \eta-2} a_{31}=A\left(y_{1} z_{2}-y_{2} z_{1}\right)\left(y_{2} z_{3}-y_{3} z_{2}\right) .
\end{array}
$$

Hiermit wird

(28.)

$$
\partial^{2 \eta+2 u-4} S=A\left|\begin{array}{lll}
z_{1} & y_{1} & k_{2} \partial^{\alpha-1} f_{3}-k_{3} \partial^{\alpha-1} f_{2} \\
z_{2} & y_{2} & k_{3} \partial^{\alpha-1} f_{1}-k_{1} \partial^{\alpha-1} f_{3} \\
z_{3} & y_{3} & k_{1} \partial^{\alpha-1} f_{2}-k_{2} \partial^{\alpha-1} f_{1}
\end{array}\right|^{2} A \cdot\left(\partial^{\alpha} f\right)^{2} \cdot k^{2} .
$$

Der Factor $A$ ist von der Ordnung $2 \eta-4$ in den z. Die Curve $S=0$ hat somit in dem betrachteten Punkte $2 \alpha$ Zweige, von deren Tangenten je zwei mit den Tangenten des Punktes von $f$ übereinstimmen, und weitere $2 \eta-4$ Zweige. Es fallen sonach in diesen Punkt $2 \alpha^{2}+2 \alpha+2 \alpha \eta-4 \alpha$ Schnittpunkte beider Curven. Die Anzahl der für unsern Zweck in Betracht kommenden Schnittpunkte, also die Anzahl der singulären Erzeugenden ist endlich:

$$
2 n(m+n-3)-\Sigma 2 \beta(\beta-1)-2 \Sigma \alpha^{2}-\Sigma\left(2 \alpha^{2}+2 \alpha+2 \alpha \eta-4 \alpha\right),
$$

wo sich die erste Summe auf die vielfachen Punkte, welche keine Ausnahmspunkte sind, die zweite auf die einfachen und die dritte auf die vielfachen Ausnahmspunkte bezieht. Die obige Zahl ist nach (16.) und (17.) gleich

$$
\text { (29.) } 2 k+4(p-1) \text {. }
$$

Diese Zahl, welche mit der, im speciellen Falle des $\$$. 5 gefundenen, übereinstimmt, gilt unter der Voraussetzung, dass die vielfachen Punkte von $f=0$ keine zusammenfallenden Tangenten besitzen, eine Voraussetzung, die aber bei allgemeinen Flächen ihrer Art stets von selbst eintreten wird.

§. 9. Die Geometrie im Strahlensystem erster Ordnung.

Als Beispiel für die in $\$$. 6 angedeutete Behandlungsweise der Geometrie im Strahlensystem, wollen wir das Strahlensystem betrachten, welches entsteht durch den Schnitt zweier linearen Complexe. 
Es seien

$$
a=\Sigma a_{i} x_{i}=0 \quad \text { und } \quad b=\Sigma b_{i} x_{i}=0
$$

die Gleichungen der beiden Complexe. Das Strahlensystem, welches sie erzeugen, ist identisch mit dem Schnitte der beiden Complexe

$$
a+\lambda_{1} b=0, \quad a+\lambda_{2} b=0 .
$$

Wir wollen nun für $\lambda_{1}$ und $\lambda_{2}$ die Wurzeln der quadratischen Gleichung setzen :

$$
\left(a_{1}+\lambda b_{1}\right)\left(a_{4}+\lambda b_{4}\right)+\left(a_{2}+\lambda b_{2}\right)\left(a_{5}+\lambda b_{5}\right)+\left(a_{3}+\lambda b_{3}\right)\left(a_{6}+\lambda b_{6}\right)=0 .
$$

Jeder der beiden letzterwähnten Complexe stellt dann eine gerade Linie dar, und wir sehen also hieraus:

Alle Strahlen des Strahlensystems, welches der Schnitt zweier linearen Complexe ist, schneiden zwei gerade Linien.

Dass diese Linien sich im Allgemeinen nicht schneiden werden ist klar. Es ist leicht, von dieser Eigenschaft ausgehend die homogenen Functionen des \$. $6 \mathrm{zu}$ bilden. Legen wir zwei gegenüberliegende Kanten des Coordinatentetraeders in die beiden Geraden, welche wir mit $A$ und $B$ bezeichnen wollen, so sind die Coordinaten eines Punktes der Linie $A$ etwa

$$
0, \quad 0, \quad a_{1}, \lambda a_{2},
$$

und eines Punktes der Linie $B$

$$
b_{1}, \quad \mu b_{2}, \quad 0, \quad 0 \text {. }
$$

Bilden wir hieraus die Coordinaten ihrer Verbindungslinie und setzen $\frac{y_{1}}{y_{3}}$ für $\lambda, \frac{y_{2}}{y_{3}}$ für $\mu$, so sind die Ausdrücke der Coordinaten $x_{1}=0, x_{2}=a_{1} b_{1} y_{3}^{2}, x_{3}=-a_{2} b_{1} y_{1} y_{3}, x_{4}=0, x_{5}=-y_{1} y_{2} a_{2} b_{2}, x_{6}=-a_{1} b_{2} y_{2} y_{3}$. Wir sehen hieraus, dass die Punkte $y_{1}=0, y_{3}=0$ und $y_{2}=0, y_{3}=0$ einfache Ausnahmspunkte sind. In dem ersten dieser Punkte wird $\frac{y_{1}}{y_{3}}$ abhängig von der Richtung, in welcher man von ihm fortgeht; es entsprechen somit den Richtungen um diesen Punkt die Linien, welche man von dem Punkte $b_{2}$ der Linie $B$ durch die Linie $A$ ziehen kann. Ebenso entsprechen dem anderen Ausnahmspunkte die Geraden, welche sich von dem Punkte $a_{2}$ der Linie $A$ durch die Gerade $B$ legen lassen.

Hieraus ist ersichtlich, dass die Linie $y_{3}=0$ in allen ihren Punkten nur die eine Linie $a_{2} b_{2}$ darstellt, die wir künftig mit $C$ bezeichnen wollen. Es ist hierbei beachtenswerth, dass $C$ nur abhängig ist von der Wahl des Coordinatentetraeders nicht aber von der Natur des Strahlensystems. 
Da zwei Linien des Systems sich nur auf einer der beiden Geraden $A, B$ schneiden können, so ist klar, dass alle in diesem System gelegenen windschiefen Flächen keine anderen vielfachen Curven als diese Geraden haben können. Es ist ferner klar, dass auch keine Doppelerzeugende im Strahlensystem existiren.

Schneiden wir nun das System durch einen Complex von $\operatorname{der} \boldsymbol{N}^{\text {ten }}$ Ordnung, so erhalten wir die der Schnittfläche entsprechende Curve, wenn wir die Werthe der $x$, wie sie oben durch die $y$ ausgedrückt sind, eintragen in die Gleichung des Complexes. Es entsteht so eine Curve $2 N^{\text {ter }}$ Ordnung, welche in den beiden Ausnahmspunkten Nfache Punkte hat.

So wird der Schnitt eines linearen Complexes dargestellt durch einen Kegelschnitt, welcher durch beide Ausnahmspunkte geht. Die entsprechende Fläche ist ein Hyperboloid. Es lässt sich leicht zeigen, dass diese Fläche unendlich viele Geraden enthält, welche alle Erzeugenden schneiden. Ist nämlich $c=0$ die Gleichung des Complexes, durch dessen Schnitt mit $a$ und $b$ sie entsteht, so liegt jede ihrer Erzeugenden auch auf dem Complexe $\lambda_{1} a+\lambda_{2} b+\lambda_{3} c=0$. Die Grössen $\lambda$ lassen sich aber auf unendlich viele Arten so bestimmen, dass dieser Complex eine gerade Linie darstellt. Hiermit ist jener bekannte Satz bewiesen.

Der Schnitt eines Complexes zweiten Grades mit dem Strahlensystem ist eine Fläche vierter Ordnung von dem Geschlechte $p=1$, welche durch eine Curve vierter Ordnung mit zwei Doppelpunkten in den Ausnahmspunkten dargestellt wird u. s. w.

Wir wollen jetzt umgekehrt untersuchen, welche Flächen durch gegebene Curven dargestellt werden. Der Grad einer Fläche, welche durch eine Curve $n^{\text {ter }}$ Ordnung, die im Ausnahmspunkte $y_{1}=0, y_{3}=0$ einen $\alpha$ fachen, im anderen einen $\beta$ fachen Punkt hat, repräsentirt wird, ist

$$
k=2 n-\alpha-\beta \text {. }
$$

Da hier jedem Werthe von $\frac{y_{1}}{y_{3}} n-\alpha$ Werthe von $\frac{y_{2}}{y_{3}}$ entsprechen, so gehen durch jeden Punkt der Linie $A \quad n-\alpha$ Erzeugende, so dass diese Linie $(n-\alpha)$ fache Curve ist. Ebenso wird $B(n-\beta)$ fache Curve. Ferner sieht man, dass jede durch $B$ gelegte Ebene $(n-\alpha)$ fache und jede Ebene durch $A(n-\beta)$ fache Tangentenebene ist. Da weiter die Curve die Linie $y_{3}=0$ noch in $n-\alpha-\beta$ Punkten schneidet, welche sämmtlich der Linie $C$ entsprechen, so ist diese Linie $(n-\alpha-\beta)$ fache Erzeugende der Fläche. 
Wenden wir dies an auf die oben betrachtete Schnittfläche des Systems mit einem Complexe $N^{\text {ier }}$ Ordnung, so folgt:

Eine windschiefe Fläche $2 N^{\text {ten }}$ Grades, welche der vollständige Schnitt zweier linearen Complexe mit einem Complex $N^{\text {ten }}$ Grades ist, hat zwei $N$ fache Curven, welche gerade Linien sind.

Wir bemerken noch, dass diese Fläche, von allen $2 N^{\text {ten }}$ Grades, welche der vollständige Schnitt dreier Complexe sind, das höchste $p$ besitzt; wie sich aus Formel (7.) \$. 3 leicht ergiebt.

Im speciellen Falle, wo $N=2$, hat die Fläche vierter Ordnung, die beiden Geraden zu Doppelcurven. Wir bemerken noch, dass diese Fläche vierten Grades die einzige ist, für welche $p=1$ ist. Denn setzen wir in (8.) $\$$. $3 k=4, p=1, d=0$ so ergiebt sich $\delta=2$, und dass diese Curve nur aus zwei sich nicht schneidenden Geraden bestehen kann, wenn die Fläche eine eigentliche Fläche sein soll, ist klar.

Einer Geraden, welche durch einen Ausnahmspunkt geht, entspricht nun ein Strahlbüschel, welches von einem Punkte der einen $\operatorname{der}$ Linien $A, B$ durch die andere gelegt ist.

Geht die Gerade nicht durch einen Ausnahmspunkt, so stellt sie ein Hyperboloid dar, welches, da die Gerade $y_{3}=0$ in einem Punkte geschnitten wird, die Linie $C$ unter seinen Erzeugenden enthält. Da eine Gerade durch zwei Punkte bestimmt und die Linie $C$ willkürlich ist, so sehen wir, dass ein Hyperboloid durch drei Erzeugende bestimmt ist.

Ein Kegelschnitt, der durch beide Ausnahmspunkte geht, ist das Bild eines Hyperboloids, welches aber die Linie $C$ nicht enthält. Da nun ein Kegelschnitt eine Curve zweiter Klasse ist, so giebt es zwei Hyperboloide, welche durch zwei Linien des Systems gehen und ein Hyperboloid, welches diese Linien nicht als Erzeugende enthält, in zwei aufeinanderfolgenden Linien schneiden.

Ein Kegelschnitt der durch einen Ausnahmspunkt geht, z. B. durch den $y_{1}=0, y_{3}=0$, stellt eine Fläche dritter Ordnung dar, welche in der Linie $B$ eine Doppel- und in $A$ eine einfache Curve besitzt. Die Linie $C$ gehört zu den Erzeugenden der Fläche. Wir sehen hieraus, dass die Fläche durch 5 ihrer Erzeugenden bestimmt ist, wenn eine Linie, welche Doppelcurve und eine andere, die einfache Curve werden soll, gegeben ist.

Geht ein Kegelschnitt durch keinen Ausnahmspunkt, so ist die ent- 
sprechende Fläche von der vierten Ordnung, $A$ und $B$ sind ihre Doppelcurven und $C$ ist eine Doppelerzeugende.

Diese Fläche erscheint als specieller Fall der allgemeinen Fläche vierter Ordnung mit den Doppelcurven $A$ und $B$, welche durch eine Curve dritter Ordnung dargestellt wird, die durch die Ausnahmspunkte geht. Für diese Fläche, welche mit der oben erwähnten identisch ist, ist $p=1$. Sie wird, wenn ihre Doppelcurven gegeben sind, durch acht Erzeugende bestimmt. Da eine Curve dritter Ordnung neun Wendepunkte hat, so giebt es neun Hyperboloide des Strahlensystems, welche durch eine gegebene Erzeugende der Fläche gehen und diese in drei auf einander folgenden Linien schneiden u. s. w.

Diese Beispiele werden hinreichen, um die Art der Uebertragung von Sätzen aus der Ebene zu zeigen und es wird leicht sein, diese Uebertragung vorzunehmen, besonders wenn man noch die projectivischen Beziehungen zwischen den windschiefen Flächen, für welche $p=0$ ist, einführt, auf welche die entsprechenden Curven, die ihre Bilder sind, hinweisen.

Mannheim, im November 1866. 